\title{
Biomedical Material
}

National Cancer Institute

\section{Source}

National Cancer Institute. Biomedical Material. NCI Thesaurus. Code C16338.

Any substance other than pharmacologic substance that can be applied in biomedical research or in constructing artificial organs, devices, or prostheses that can be introduced into the human body. 